\title{
Critical role of PET/CT-based novel quantitative techniques for assessing global disease activity in multiple myeloma and other hematological malignancies: why it is time to abandon reliance on examining focal lesions
}

\author{
Raheleh Taghvaei $^{1} \cdot$ Mahdi Zirakchian Zadeh ${ }^{1,2} \cdot$ Thomas J. Werner $^{1} \cdot$ Abass Alavi $^{1}$ \\ Received: 12 August 2020 / Revised: 17 September 2020 / Accepted: 12 October 2020 / Published online: 30 October 2020 \\ (C) European Society of Radiology 2020
}

PET/CT and PET/MRI have emerged as useful modalities in many domains including those of malignant disorders. The recent introduction of a PET instrument with 2 meters field of view allows imaging the entire body simultaneously within a short period of time. These innovations will allow assessing disease that are diffuse in nature and are known to involve many organs and structures throughout the body. While most solid tumors are confined to the bones and organs in the trunk, hematologic malignancies including multiple myeloma (MM) are known to be widespread and involve the entire skeleton. Therefore, imaging the entire body is essential for a comprehensive assessment of the affected population.

Structural imaging techniques are mostly employed as qualitative modalities and are therefore of limited value in monitoring the course of the disease. In contrast, PET imaging by its nature is a quantitative modality, and as such, provides precise degrees of disease activity in the lesions visualized. Therefore, quantification by PET is of great importance in the management of numerous diseases and disorders that are encountered in the day to day practice of medicine. This unique

This comment refers to the article available at https://doi.org/10.1007/ s00330-020-07177-x.

Abass Alavi

abass.alavi@pennmedicine.upenn.edu

Thomas J. Werner

Tom.Werner@pennmedicine.upenn.edu

1 Department of Radiology, Hospital of the University of Pennsylvania, 3400 Spruce St, Philadelphia, PA 19104, USA

2 Department of Radiology, Children's Hospital of Philadelphia, 3401 Civic Center Boulevard, Philadelphia, PA 19104, USA capability of PET is essential for early detection as well as monitoring the course of hematologic cancers including MM.

Increasingly, PET imaging is employed in the management of MM. Based on the Revised International Myeloma Working Group (IMWG) diagnostic criteria, presence of more than one focal bone lesion on MRI and one or more osteolytic lesions on skeletal x-ray, CT, or FDG-PET/CT should be considered for initiation of treatment [1]. The Italian Myeloma criteria for PET Use (IMPeTUs) suggest visual interpretation of FDG-PET/CT including bone marrow metabolic activity based on Deauville five-point scale, number and site of FDG-avid focal lesions, number of osteolytic lesions, and extramedullary disease (EMD) [2]. Several findings on FDG-PET scan have been recognized as predictive factors in MM, including the SUVmax, more than 3 focal lesions, and presence of EMD [3, 4].

Unfortunately, the existing data suffer from serious shortcomings and need to be clarified and redefined for optimal applications of this powerful technology in the management of the hematological malignancies. Quantitative techniques with PET are complicated and every effort should be made for its optimal utilization in such complicated settings. Compared to CT and MRI, PET generates images with a relatively low spatial resolution because of the physical nature of this modality. While CT and MRI can detect structures that are smaller than a millimeter, the spatial resolution of PET is 8-10 $\mathrm{mm}$ in human body imaging. This poses a major challenge in detecting and quantifying abnormalities that are a fraction of a centimeter in size in many organs of the body including the bone marrow. Also, the limited spatial resolution (the socalled partial volume effect (PVE)) of PET leads to underestimation of disease activity in focal lesions and must be corrected for generating accurate values [5]. Measuring SUVmax is 
particularly of limited importance, since it only reflects measurements from a limited sample of the disease sites and can be misleading [6]. As such, the data that are based on SUVmax cannot be extrapolated to the entire lesion and considered as being representative of the overall disease activity. Therefore, the validity of the claims made based on SUVmax measurements is questionable. Furthermore, as noted above, the hematologic malignancies are diffuse in nature, and as a result, the degree and the extent of the disease activity should be assessed throughout the skeleton and throughout the body. We believe the validity of making clinical decisions based on assessment of only three or several focal lesions is questionable, since MM infiltrates the bone marrow diffusely, and therefore, this approach should be abandoned in the future.

In order to overcome the deficiencies described, global assessment based on SUVmean should be adopted as the technique of choice in the future [7]. Recently introduced CT-based segmentation techniques allow for practical application of this novel approach in any setting where PET/CT imaging is routinely performed for both clinical and research purposes. The software (OsiriX software, Pixmeo SARL) provides global values by a $3 \mathrm{D}$ growing region algorithm with a lower and upper threshold of 150-1500 Hounsfield units followed by a morphological closing algorithm with a structuring element radius of 20 applied to the fused PET/CT images. The segmented images include both the cortical and trabecular bony structures which lead to generating global
SUVmean (G SUVmean) measurement for the entire skeleton (Fig. 1). In a study based on this technique, G SUVmean of 21 patients with MM before and after treatment was compared to the control group. The results showed that the $\mathrm{G}$ SUVmean in patients before treatment is significantly higher than the control group. After treatment, there was no significant difference between the G SUVmean of the patient and the control groups [8]. This assessment method has also been applied for measuring bone metabolism by $18 \mathrm{~F}$-sodium fluoride $\mathrm{PET} / \mathrm{CT}$, which has revealed a significant drop in $\mathrm{G}$ SUVmean in patients with MM after high-dose chemotherapy [9]. We believe, G SUVmean measurements will be of great value in detecting and quantifying EMD activity in MM [10].

Finally, we should point out that in addition to its ability to detect cancer, FDG-PET imaging allows for determining the degree of aggressiveness and the inhomogeneity of various lesions throughout the body. By now, it is well established that malignant lesions show increasing uptake of FDG over several hours. In contrast, normal tissues or benign abnormalities with the initial FDG uptake reveal substantial washout over time. Adopting this approach will further enhance the specificity of FDG-PET imaging in this population [11]. This methodology also will be of great value for assessing frequent but non-specific abnormities that are encountered on structural imaging techniques. While the role of FDGPET is very well established in multiple myeloma and
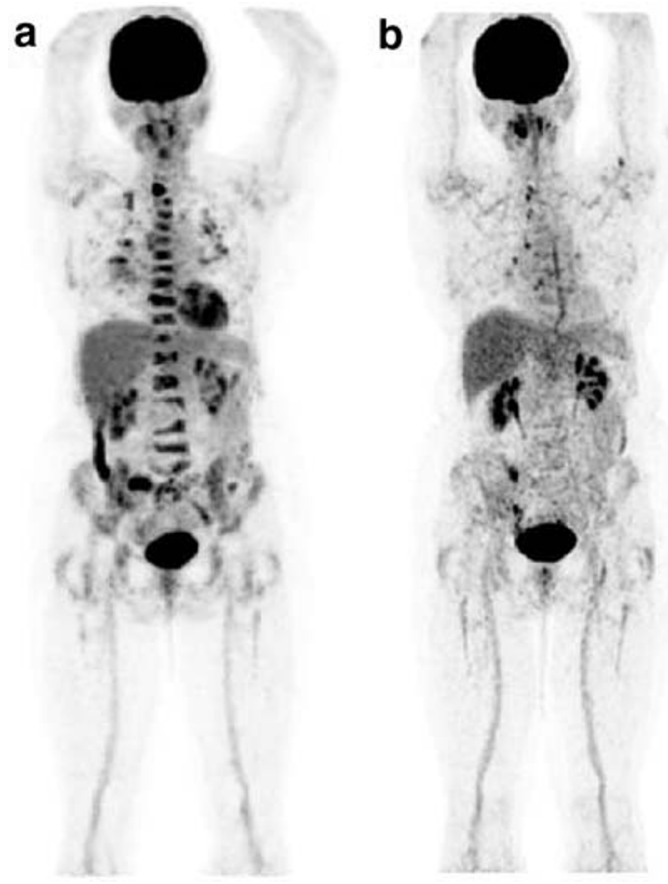

Fig. 1 Potential role of global disease assessment by PET in MM. FDG uptake changes of MM lesions before (a) and after the treatment (b). High diffuse FDG uptake is observed in the entire spine before the treatment (a), whereas substantial reduction in FDG uptake is visually noted after the treatment (b). Segmentation of the entire skeleton followed by a
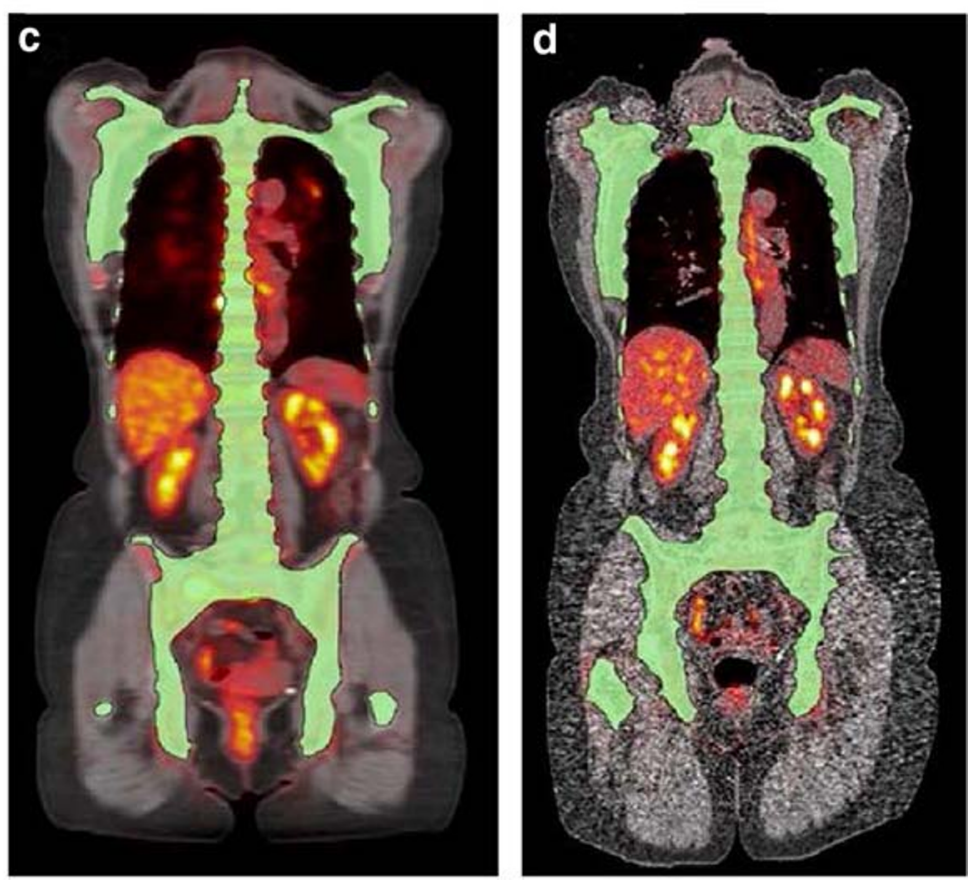

closing algorithm allows for one to perform global disease assessment (OsiriX software, Pixmeo SARL) (c, d). The pretreatment global average SUVmean (c) was 3.1 and decreased to 1.8 after the completion of the treatment (d) (reprinted with permission from Zadeh MZ et al [7]) 
lymphoproliferative disorders, its application in other hematological malignancies is unclear at this time.

Therefore, PET-CT imaging has a great promise for the management of patients with MM and other hematologic malignancies and should be used as the imaging modality of choice in such settings. However, every effort should be made to avoid potential sources of error in quantifying disease activity in the bone marrow and the other organs in the body with this powerful modality.

Funding The authors state that this work has not received any funding.

\section{Compliance with ethical standards}

Guarantor The scientific guarantor of this publication is Prof. Abass Alavi.

Conflict of interest The authors of this manuscript declare no relationships with any companies whose products or services may be related to the subject matter of the article.

Statistics and biometry No complex statistical methods were necessary for this paper.

Informed consent Approval from the institutional animal care committee was not required because it was not conducted on human subjects.

Ethical approval Institutional Review Board approval was not required because this article is an editorial.

\section{Methodology}

- This is an editorial article.

\section{References}

1. Rajkumar SV, Dimopoulos MA, Palumbo A et al (2014) International Myeloma Working Group updated criteria for the diagnosis of multiple myeloma. Lancet Oncol 15(12):e538-e548
2. Nanni C, Zamagni E, Versari A et al (2016) Image interpretation criteria for FDG PET/CT in multiple myeloma: a new proposal from an Italian expert panel. IMPeTUs (Italian Myeloma criteria for PET USe). Eur J Nucl Med Mol Imaging 43(3):414-421

3. Zamagni E, Patriarca F, Nanni C et al (2011) Prognostic relevance of 18-F FDG PET/CT in newly diagnosed multiple myeloma patients treated with up-front autologous transplantation. Blood 118(23):5989-5995

4. Haznedar R, Ak1 SZ, Akdemir ÖU et al (2011) Value of 18 Ffluorodeoxyglucose uptake in positron emission tomography/ computed tomography in predicting survival in multiple myeloma. Eur J Nucl Med Mol Imaging 38(6):1046-1053

5. Alavi A, Werner TJ, Høilund-Carlsen PF, Zaidi H (2018) Correction for partial volume effect is a must, not a luxury, to fully exploit the potential of quantitative PET imaging in clinical oncology. Mol Imaging Biol 20(1):1-3

6. Kwee TC, Cheng G, Lam MGEH, Sandip B, Alavi A (2013) SUVmax of 2.5 should not be embraced as a magic threshold for separating benign from malignant lesions. Eur J Nucl Med Mol Imaging 40:1475-1477

7. Zadeh MZ, Raynor WY, Seraj SM et al (2019) Evolving roles of fluorodeoxyglucose and sodium fluoride in assessment of multiple myeloma patients: introducing a novel method of PET quantification to overcome shortcomings of the existing approaches. PET Clin 14(3):341-352

8. Zadeh MZ, Raynor W, Oestergaard B et al (2018) Changes in bone marrow FDG uptake in multiple myeloma patients before and after treatment. J Nucl Med 59:1430-1430

9. Zadeh MZ, Østergaard B, Raynor WY, et al (2020) Comparison of $18 \mathrm{~F}$-sodium fluoride uptake in the whole bone, pelvis, and femoral neck of multiple myeloma patients before and after high-dose therapy and conventional-dose chemotherapy. Eur J Nucl Med Mol Imaging 47 (12):2846-2855

10. Zadeh MZ, Nguyen D, Ostergaard B et al (2019) Baseline global splenic uptake of FDG in multiple myeloma patients: a higher uptake is associated with inferior overall survival. J Nucl Med 60:2222

11. Taghvaei R, Østergaard B, Zadeh MZ et al (2017) Correlation of dual time point FDG-PET with response to chemotherapy in multiple myeloma. J Nucl Med 58:188-188

Publisher's note Springer Nature remains neutral with regard to jurisdictional claims in published maps and institutional affiliations. 